\section{Personalization of loco-regional care for primary breast cancer patients (part 1)}

\author{
Masakazu Toi*, Eric P Winer, John R Benson, Takashi Inamoto, John F Forbes, \\ Gunter von Minckwitz, John FR Robertson, Stephen R Grobmyer, Ismail Jatoi, \\ Hironobu Sasano, Ian Kunkler, Alice Y Ho, Chikako Yamauchi, Louis WC Chow, \\ Chiun-Sheng Huang, Wonshik Han, Shinzaburo Noguchi, Mark D Pegram, \\ Hideko Yamauchi, Eun-Sook Lee, Alexey A Larionov, Jose LB Bevilacqua, \\ Michio Yoshimura, Tomoharu Sugie, Akira Yamauchi, lan E Krop, Dong Young Noh, \\ V Suzanne Klimberg \& 2014 Kyoto Breast Cancer Consensus Conference
}

\section{Kyoto Breast Cancer Consensus Conference, Kyoto, Japan, 18-20 February 2014}

The loco-regional management of breast cancer is increasingly complex with application of primary systemic therapies, oncoplastic techniques and genetic testing for breast cancer susceptibility. Personalization of loco-regional treatment is integral to optimization of breast cancer care. Clinical and pathological tumor stage, biological features and host factors influence loco-regional treatment strategies and extent of surgical procedures. Key issues including axillary staging, axillary treatment, radiation therapy, primary systemic therapy (PST), preoperative hormonal therapy and genetic predisposition were identified and discussed at the Kyoto Breast Cancer Consensus Conference (KBCCC2014). In the first of a two part conference scene, consensus recommendations for axillary management are presented and focus on the following topics: indications for completion axillary lymph node dissection in primary surgical patients with $\leq 2$ macrometastases or any sentinel nodal deposits after PST; the timing of sentinel lymph node biopsy in the context of PST; use of axillary irradiation as a component of primary treatment plans and the role of intraoperative node assessment in the post-Z0011 era.

The American Society of Clinical Oncology clinical practice guideline recommends that women without sentinel lymph node metastases should not receive axillary lymph node dissection (ALND) [1]. Recent randomized trials have examined noninferiority of sentinel lymph node (SLN) biopsy alone in comparison to SLN biopsy followed by either completion ALND or axillary radiotherapy (ART) in clinically node-negative patients with one or two nodes positive for micro/macrometastases and receiving standard schedules of adjuvant treatments. It seems likely that completion ALND can be safely omitted in selected patients, although longer term follow-up data are essential $[2,3]$. The concept of SLN biopsy being therapeutic is gaining widespread acceptance within the breast cancer community and influencing routine practice [4]. Patient selection criteria used within the Z0011 trial are being applied with variable degrees of stringency - recent surveys suggest that up to one-quarter of breast units are still undertaking completion ALND for micrometastases only [5]. Amongst those patients with limited node involvement who are treated with breast conserving

*Author for correspondence: Breast Surgery, Graduate School of Medicine, Kyoto University, 54 Shogoin Kawara-cho, Sakyo-ku,

Kyoto 606-8507, Japan; toi@kuhp.kyoto-u.ac.jp

For a full list of affiliations, please see page 1300

\section{KEYWORDS}

- axillary conserving therapy - breast cancer • locoregional care $\bullet$ preoperative systemic therapy $\bullet$ sentinel lymph node biopsy 
surgery (BCS) and systemic therapy, SLN biopsy alone does not lead to inferior survival outcomes in terms of regional control, disease-free nor overall survival compared with SLN biopsy and completion ALND. The latest American Society of Clinical Oncology guidelines state that women with one or two metastatic sentinel nodes following BCS with whole breast irradiation and systemic therapy can avoid completion ALND [1]. There remains uncertainty about precise details of radiation fields to the lower axilla and further information is awaited. There was consensus agreement at Kyoto Breast Cancer Consensus Conference that completion ALND should not be recommended for patients with SLN micrometastases only. The panel acknowledged that approximately $50 \%$ of breast surgeons have embraced results of the ACOSOG Z0011 trial and modified axillary surgery for BCS patients with a low axillary tumor burden ( $\leq 2$ sentinel lymph node macrometastases). It remains unclear whether primary mastectomy patients can safely avoid ALND for $\leq 2$ sentinel nodes containing macrometastases. Neither the Z0011 nor IBCSG 23-01 trials specifically addressed this issue although the AMAROS trial randomized patients with both partial and total mastectomy to ALND or radiation therapy $[2-3,6-7]$. By contrast, the POSNOC trial randomizes mastectomy and $\mathrm{BCS}$ patients with one or two macrometastatic nodes to either no further surgery or completion ALND [8]. In the AMAROS trial, the sentinel node was positive in $51 \%$ of patients with multifocal tumors and in $28 \%$ of patients with unifocal lesions; additional nonsentinel lymph node metastases were found in $40 \%$ of the multifocal group and in $39 \%$ of the unifocal disease group [6]. Both completion ALND (to level III) and ART following SLN biopsy provided equivalent regional disease control, but ART was associated with much lower rates of lymphedema. Therefore both axillary dissection and radiotherapy provide good locoregional control for node-positive patients, but radiotherapy is associated with lower rates of lymphedema $(21.7$ vs $40 \%$; p < 0.001$)$ in shortterm follow-up. KBCCC2014 highlighted a hybrid procedure termed "conservative axillary regional excision" (CARE) which has been described by Japanese surgeons and involves removal of tracer sentinel and other palpably suspicious lymph nodes. Neither intraoperative frozen section nor reoperation for node-positive cases was undertaken as part of CARE and the regional recurrence rate among a group of $\mathrm{pN} 1$ patients was relatively low (3.4\%) [7]. Those patients with limited metastatic tumor burden (1 or 2 sentinel nodes with macrometastases; metastatic ratio $\leq 0.5$ ) would be suitable for ACT as part of contemporaneous practice. The presence of extra-nodal spread or extensive infiltration of 3 or more sentinel nodes would warrant more intensive regional therapy with completion ALND and supraclavicular nodal irradiation/ internal mammary nodal irradiation.

An important question is whether or not ACT (be this SLN biopsy alone or combined with ART) is appropriate for patients who have undergone mastectomy and for whom post-mastectomy radiotherapy (PMRT) is planned. In the AMAROS trial, both partial and total mastectomy cases with a positive SLN biopsy were randomized to ALND or radiation therapy to the axillary and supraclavicular nodal basins. There was no significant difference for either regional disease control or overall survival suggesting that radiation therapy can effectively suppress locoregional disease recurrence in selected patients with low volume nodal disease [6]. Since PMRT is indicated for some node-positive patients, ACT could be evaluated among (total) mastectomy patients to reduce rates of lymphedema. Contemporary analyses have demonstrated how axillary recurrence has decreased dramatically consequent to advances in systemic treatment and radiation therapy, suggesting that ACT might be applicable to patients responding well to PST [4].

Pathological nodal status and breast tumor response following PST predicts for locoregional recurrence and guides use of adjuvant radiotherapy $[8,9]$. Patient selection and timing of SLN biopsy in the context of PST remains controversial; SLN biopsy undertaken prior to PST minimizes risk of a false negative result and may allow more accurate pathological staging $[10,11]$. Knowledge of nodal response to PST provides prognostic information, although it is uncertain whether PMRT should be based upon pathological nodal status after PST as well as clinical stage at presentation. Whether response to PST can select those patients most likely to benefit from PMRT is unknown [12]. The National Surgical Adjuvant Breast and Bowel Project B-32 trial confirmed high rates of identification for SLN biopsy in clinically node-negative disease $(92.6 \%)$ with an overall false-negative rate (FNR) of $9.8 \%$ [11]. However, 
the ACOSOG Z1071 (Alliance) trial reported FNR of $12.6 \%$ among women with clinically N1 disease receiving PST who had two or more sentinel nodes examined [12]. In the German SENTINA study, patients who converted after PST from $\mathrm{cN}+$ to ycN0, had detection rates of $80.1 \%$ while the FNR was $14.2 \%$, similar to Z1071 for this category of patients. However, the FNR was much higher (24.3\%) when only a single node was removed and $51.6 \%$ in patients who had a second SLN biopsy procedure after PST [13]. In clinically node-positive patients who had SLN biopsy after PST, the false negative rate decreased to $8.6 \%$ with dual tracer methodology (blue dye and radioisotope) and at least three nodes dissected. When nodal involvement is proven by core biopsy/fine needle aspiration cytology or strongly suspected by sonographic features, SLN biopsy should be considered after PST if a favorable tumor response is anticipated (pCR or near pCR). Removal of more than two sentinel nodes improves accuracy of SLN biopsy in clinically N1 patients following PST. Several ongoing clinical trials are assessing the role of post-PST SLN biopsy (details are not shown). There was discussion at Kyoto Breast Cancer Consensus Conference of whether fluorescence imaging with indocyanine green can offer advantages for detection of SLNs after PST [14-16]. For clinically and sonographically node-negative patients, SLN biopsy before PST should be considered for staging purposes and can influence decision-making for systemic therapies and radiation therapy. However, it is acceptable practice to undertake SLN biopsy either before or after PC and should involve dual localization techniques (blue dye and isotope) when core biopsy positive at presentation [13].
A positive SLN after PC (isolated tumor cells, micro-/macrometastases) should always prompt completion ALND which is also preferable in those patients who are SLN negative after PC but node positive initially. Intraoperative node assessment may become less relevant in the post-Z0011 era when increasing numbers of SLN positive patients receive no further axillary treatment (surgery or radiotherapy). There was general agreement that molecular thresholds for distinguishing between micro- and macro-metastases may have limited accuracy [17].

\section{Acknowledgements}

The authors sincerely thank professor Masahiro Hiraoka, Kyoto University. The authors deeply thank Dr Sung Won Kim, Dr Seigo Nakamura, Dr Shinji Ohno, Dr Hiroji Iwata, Dr Takayuki Kinoshita, Dr Chieko Tamura, Dr Naoto Shikama, Dr Norikazu Masuda, Dr Toshikazu Ito, Dr Hiroko Masuda, Dr Masahiro Sugimoto, Dr Shotaro Kanao and Dr Masako Kataoka and Dr Shigehira Saji. The authors are also grateful to Dr Hiroshi Ishiguro and Dr Masahiro Takada. The authors thank Ms Aya Morotomi, Ms Mihoko Yamamoto and Ms Chisa Takano and Mr David Graham for their help. The authors thank The Uehara Memorial Foundation, The Naito Foundation, Yamaguchi Endocrine Research Foundation and Kyoto Convention Bureau.

\section{Financial \& competing interests disclosure}

The authors acknowledge the kind support of the Japan Breast Cancer Society. The authors have no other relevant affiliations or financial involvement with any organization or entity with a financial interest in or financial conflict with the subject matter or materials discussed in the manuscript apart from those disclosed.

No writing assistance was utilized in the production of this manuscript.

\section{References}

1 Lyman GH, Temin S, Edge SB et al. Sentinel lymph node biopsy for patients with early-stage breast cancer: American Society of Clinical Oncology Clinical Practice Guideline Update. J. Clin. Oncol. 32(13), 1365-1383 (2014).

2 Giuliano AE, Hunt KK, Ballman KV et al. Axillary dissection vs no axillary dissection in women with invasive breast cancer and sentinel node metastasis: a randomized clinical trial. JAMA 305(6), 569-575 (2011).

3 Galimberti V, Cole BF, Zurrida S et al. International Breast Cancer Study Group Trial 23-01 investigators. Axillary dissection versus no axillary dissection in patients with sentinel-node micrometastases (IBCSG 23-01): a Phase 3 randomised controlled trial. Lancet Oncol. 14(4), 297-305 (2013).

4 Morrow M. It is not always necessary to do axillary dissection for $\mathrm{T} 1$ and $\mathrm{T} 2$ breast cancer - point. Cancer Res. 73(24), 7151-7154 (2013).

5 Dengel LT, Van Zee KJ, King TA et al. Axillary dissection can be avoided in the majority of clinically node-negative patients undergoing breast-conserving therapy. Ann. Surg. Oncol. 21(1), 22-27 (2014).

6 Donker M, van Tienhoven G, Straver ME et al. Radiotherapy or surgery of the axilla after a positive sentinel node in breast cancer
(EORTC 10981-22023 AMAROS): a randomised, multicentre, open-label, Phase 3 non-inferiority trial. Lancet Oncol. 15(12), 1303-1310 (2014).

7 Cowher MS, Grobmyer SR, Lyons J et al. Conservative axillary surgery in breast cancer patients undergoing mastectomy: long-term results. J. Am. Coll. Surg. 218(4), 819-824 (2014).

8 Mamounas EP, Anderson SJ, Dignam JJ et al. Predictors of locoregional recurrence after neoadjuvant chemotherapy: results from combined analysis of National Surgical Adjuvant Breast and Bowel Project B-18 and B-27. J. Clin. Oncol. 30(32), 3960-3966 (2012). 
9 Benson JR, Jatoi I. Sentinel lymph node biopsy and neoadjuvant chemotherapy in breast cancer patients. Future Oncol. 10(4), 577-586 (2014).

10 Vaz-Luis I, Winer EP, Lin NU. Human epidermal growth factor receptor-2-positive breast cancer: does estrogen receptor status define two distinct subtypes? Ann. Oncol. 24(2), 283-291 (2013).

11 Harlow SP, Krag DN, Julian TB et al. Prerandomization Surgical Training for the National Surgical Adjuvant Breast and Bowel Project (NSABP) B-32 trial: a randomized Phase III clinical trial to compare sentinel node resection to conventional axillary dissection in clinically node-negative breast cancer. Ann. Surg. 241(1), 48-54 (2005).

\section{Affiliations}

\section{- Masakazu Toi}

Breast Surgery, Graduate School of Medicine, Kyoto University, 54 Shogoin Kawara-cho, Sakyo-ku, Kyoto 606-8507, Japan

- Eric P Winer Breast Oncology Center, Dana-Farber Cancer Institute, Brigham \& Women's Cancer Center, Harvard Medical School, Boston, MA 02215, USA

- John R Benson Cambridge Breast Unit, Addenbrooke's Hospital \& University of Cambridge, Cambridge, UK

- Takashi Inamoto Faculty of Health Care, Tenri Health Care University, Nara, Japan

- John F Forbes Surgical Oncology, University of Newcastle, Newcastle, Australia

- Gunter von Minckwitz German Breast Group, Frankfurt, Germany

- John FR Robertson Breast Surgery, University of Nottingham, Royal Derby Hospital, Nottingham, UK

- Stephen R Grobmyer Cleveland Clinic \& Lerner College of Medicine of Case Western Reserve University, Cleveland, OH 44195, USA

- Ismail Jatoi The University of Texas Health Science Center, San Antonio, TX 78229, USA
12 Boughey JC, Suman VJ, Mittendorf EA et al. Sentinel lymph node surgery after neoadjuvant chemotherapy in patients with node-positive breast cancer: the ACOSOG Z1071 (Alliance) clinical trial. JAMA 310 (14), 1455-1461 (2013).

13 Kuehn T, Bauerfeind I, Fehm T et al. Sentinel-lymph-node biopsy in patients with breast cancer before and after neoadjuvant chemotherapy (SENTINA): a prospective, multicentre cohort study. Lancet Oncol. 14(7), 609-618 (2013).

14 Wishart GC, Loh SW, Jones L, Benson JR. A feasibility study (ICG-10) of indocyanine green (ICG) fluorescence mapping for sentinel lymph node detection in early breast cancer. Eur. J. Surg. Oncol. 38(8), 651-656 (2012).

\section{- Hironobu Sasano}

Pathology, Tohoku University School of Medicine, Sendai, Japan

- Ian Kunkler Edinburgh Cancer Research UK Centre, The University of Edinburgh, UK

\section{- $\quad$ Alice Y Ho}

Radiation Oncology, Memorial SloanKettering Cancer Center, NY 10065, USA

- Chikako Yamauchi Radiation Oncology, Shiga Medical Center for Adults, Moriyama, Japan

- Louis WC Chow UNIMED Medical Institute/University of Hong Kong, Hong Kong

- Chiun-Sheng Huang Surgery, National Taiwan University Hospital, College of Medicine, National Taiwan University, Taipei, Taiwan

- Wonshik Han Surgery, Seoul National University Hospital, Seoul, South Korea

- Shinzaburo Noguchi Breast \& Endocrine Surgery, Graduate School of Medicine, Osaka University, Osaka, Japan

\section{- Mark D Pegram} Stanford Cancer Institute, School of Medicine, Stanford University, CA 94305, USA

- Hideko Yamauchi Department of Surgery, St Lukes Hospital, Tokyo, Japan
15 Sugie T, Sawada T, Tagaya N et al. Comparison of the indocyanine green fluorescence and blue dye methods in detection of sentinel lymph nodes in early-stage breast cancer. Ann. Surg. Oncol. 20(7), 2213-2218 (2013).

16 Ballardini B, Santoro L, Sangalli C et al. The indocyanine green method is equivalent to the ${ }^{99} \mathrm{mTc}$-labeled radiotracer method for identifying the sentinel node in breast cancer: a concordance and validation study. Eur. J. Surg. Oncol. 39(12), 1332-1336 (2013).

17 Benson JR, Wishart GC. Is intra-operative nodal assessment essential in a modern breast practice? Eur. J. Surg. Oncol. 36(12), 1162-1164 (2010).

- Eun-Sook Lee Surgery, Seoul National University Hospital, Seoul, South Korea

- Alexey A Larionov Statistics \& Computational Biology Laboratory, University of Cambridge, Cancer Research UK Cambridge Institute, UK

- Jose LB Bevilacqua Department of Breast Surgery, AC Camargo Cancer Center, Sao Paulo, Brazil

- Michio Yoshimura Radiation Oncology, Graduate School of Medicine, Kyoto University, Kyoto, Japan

- Tomoharu Sugie Beast Surgery, Kansai Medical University, Osaka, Japan

- Akira Yamauchi Breast Center, Kitano Hospital, The Tazuke Kofukai Medical Research Institute, Osaka, Japan

- Ian E Krop Breast Oncology Center, Dana-Farber Cancer Institute, Brigham \& Women's Cancer Center, Harvard Medical School, Boston, MA 02215, USA

- Dong Young Noh Surgery, Seoul National University Hospital, Seoul, South Korea

- V Suzanne Klimberg Surgery, Rockefeller Cancer Institute, University of Arkansas for Medical Sciences, AR 72205, USA 